\title{
Um Exercício de Priorização e Busca por Consenso Usando Planos de Ação Nacional para a Conservação de Espécies Ameaçadas no Brasil
}

\author{
Emanuelle Cordeiro de Azevedo Souza ${ }^{1,2,3}$ \& Enrico Bernard ${ }^{1}$
}

Recebido em 06/08/2020 - Aceito em 02/12/2020

1 Laboratório de Ciência Aplicada à Conservação da Biodiversidade, Departamento de Zoologia, Universidade Federal de Pernambuco. Rua Nelson Chaves s/n, Cidade Universitária, Recife/PE, Brasil. CEP: 50.670-901. <emanuelle@cepan.org.br, enricob2@gmail.com>.

2 Programa de Pós-Graduação em Biologia Animal, Departamento de Zoologia, Universidade Federal de Pernambuco. Rua Nelson Chaves s/n, Cidade Universitária, Recife/PE, Brasil. CEP: 50.670-901. <emanuelle@cepan.org.br>

3 Centro de Pesquisas Ambientais do Nordeste/CEPAN. Av. Montevidéu, 172, salas 1105 e 1106, Bairro Boa Vista, Recife/PE, Brasil. CEP: 50.050-250.<emanuelle@cepan.org.br>.

RESUMO - Diante da urgência e de múltiplos alvos para a conservação da biodiversidade, e a escassez de recursos financeiros e vontade política, é importante o consenso sobre quais ações devem ser prioritárias. Técnicas multicritérios podem contribuir para a melhoria do processo de priorização em conservação e diferentes iniciativas e abordagens poderiam se beneficiar de seu uso. Apresentamos aqui um exercício de priorização e busca de consenso a partir da utilização de uma técnica multicritério para ranquear os objetivos propostos por três planos de ação nacional no Brasil (PANs). Esse ranqueamento permitiu: 1) comparar se a ordenação das ações nesses PAN corresponde às ações consideradas prioritárias pelo público que elaborou este documento; 2) identificar idiossincrasias entre o que diz um documento oficial de política pública e o que pensam as pessoas responsáveis pela execução dessas ações; e 3) identificar ações prioritárias comuns a mais de um plano - e que podem otimizar o processo de conservação da biodiversidade. Houve falta de congruência entre os objetivos ordenados nos planos de ação e o ranking de prioridades feito pelos especialistas que elaboraram estes mesmos planos. Um conjunto de objetivos comuns foi identificado como prioridade em mais um plano de ação. Entretanto, nem sempre estes objetivos comuns foram prioritários em seus respectivos planos de ação. Alertamos que a falta de congruência observada pode comprometer a execução de alguns dos objetivos gerais, prejudicando ou até inviabilizando a conservação das espécies-alvo desses documentos.

Palauras-chave: Análises multicritérios; conservação da biodiversidade; efetividade na conservação; espécies ameaçadas; políticas ambientais.

\section{An Exercise of Conservation Prioritization and Search of Consensus Using Species Action Plans in Brazil}

\begin{abstract}
In view of the urgency and multiple targets for biodiversity conservation, and the scarcity of financial resources and political will, it is important to reach a consensus on which actions should be given priority. Multicriteria techniques can contribute to the improvement of the conservation prioritization process and different initiatives and approaches could benefit from their use. Here we present an exercise in prioritizing and seeking consensus using a multicriteria technique to rank the objectives proposed by three national action plans in Brazil. This ranking allowed: 1) to compare whether the order of appearance of the actions in these plans are in fact considered to be priorities by the public that drafted these documents; 2) to identify idiosyncrasies between what an official public policy document says and what the people responsible for execution think of these actions; and 3) to identify priority actions common to more than one plan - which can optimize the biodiversity conservation process. We identified a lack of congruence between the order of objectives identified in the action plans and the ranking of priorities made by the specialists who prepared these same plans. A set of common objectives was identified as a priority among different Plans. However, these common objectives were not always a priority in their respective Action Plans. We warn that the lack of congruence observed can compromise the execution of some of the general objectives, humpering or even preventing the conservation of the target species of these documents.
\end{abstract}

Keywords: Biodiversity conservation; conservation effectiveness; endangered species; environmental policies; multicriteria analysis. 


\section{Un Ejercicio de Priorización y Búsqueda de Consenso Utilizando Planes de Acción Nacionales para la Conservación de Especies Amenazadas en Brasil}

RESUMEN - Dada la urgencia y los múltiples objetivos para la conservación de la biodiversidad, y la escasez de recursos financieros y voluntad política, es importante llegar a un consenso sobre qué acciones deben tener prioridad. Las técnicas multicriterio pueden contribuir a la mejora del proceso de priorización de la conservación y diferentes iniciativas y enfoques podrían beneficiarse de su uso. Aquí presentamos un ejercicio de priorización y búsqueda de consensos utilizando una técnica multicriterio para clasificar los objetivos propuestos por tres planes de acción nacionales en Brasil. Este ranking permitió 1) comparar si el ordenamiento de las acciones en estos planes corresponde a las acciones consideradas prioritarias por el público que elaboró este documento, 2) identificar idiosincrasias entre lo que dice un documento oficial de política pública y lo que piensan los responsables de llevar a cabo estas acciones y 3) identificar acciones prioritarias que sean comunes a más de un plan y que puedan optimizar el proceso de conservación de la biodiversidad. Hubo una falta de congruencia entre los objetivos ordenados en los planes de acción y la jerarquización de prioridades realizada por los especialistas que elaboraron estos mismos planes. Un conjunto de objetivos comunes fue identificado como una prioridad en otros planes de acción. Sin embargo, estos objetivos comunes no siempre fueron una prioridad en sus respectivos planes de acción. Advertimos que la falta de congruencia observada puede comprometer la ejecución de algunos de los objetivos generales, perjudicando o incluso impediendo la conservación de las especies-objetivo de estos documentos.

Palabras clave: Análisis multicriterio; conservación de la biodiversidad; efectividad de la conservación; especies en peligro; políticas ambientales.

\section{Introdução}

Em um cenário de múltiplos alvos e de urgência conservacionista, é importante que haja um consenso entre as partes interessadas para que se alcance o sucesso no exercício da conservação, pois em caso contrário as discordâncias podem resultar em barreiras para a execução das ações (Crees et al., 2015; Gregory et al., 2012; Jacobs et al., 2014; McShane et al., 2011). Esse consenso em conservação é também desejável considerando a escassez de recursos financeiros $e$ a existência de contextos sociais, econômicos e políticos frequentemente contrários à conservação da biodiversidade. A redução das distâncias entre a teoria e a prática, a remoção de barreiras para a ação e a busca por consenso são abordados pela Decision Science, um campo da Ciência que mistura matemática aplicada, economia, filosofia e psicologia e tem por finalidade ajudar as pessoas na tomada de decisões, principalmente quando elas são complexas e incertas, características frequentes na área da Conservação da Biodiversidade (Game et al., 2013).

Os processos de tomada de decisão somados aos de resolução de conflitos em conservação são frequentemente longos e custosos e requerem forte investimento de energia para a solução de problemas, podendo ainda, se não resolvidos, ter consequências negativas tanto para a conservação quanto para o desenvolvimento econômico e social (Redpath et al., 2013). Para tornar os processos de tomada de decisão viáveis, existem diferentes alternativas, entre as quais estão técnicas multicritério, importantes por serem capazes de resolver problemas cujas avaliações são conflitantes (Ananda \& Herath, 2003; Yavuz \& Baycan, 2013). Uma dessas técnicas é a Análise Hierárquica de Processos (AHP), que permite que o tomador de decisões solucione o problema com base em suas experiências próprias e é comumente utilizada em decisões que envolvem múltipla escolha de critérios, promovendo uma estrutura para a seleção de preferências em meio a uma série de potenciais soluções (Chow \& Sadler, 2010; Yavuz \& Baycan, 2013; Govindan et al., 2015).

Se bem aplicadas, as técnicas multicritérios podem contribuir para a melhoria do processo de identificação de prioridades em conservação $e$ diferentes iniciativas e abordagens poderiam se beneficiar de seu uso. Um exemplo são os planos de ação nacional para a conservação das espécies ameaçadas de extinção (Species Action Plans, daqui em diante PANs), parte das estratégias e planos de ação nacional para biodiversidade (National Biodiversity Strategies and Action Plans - NBSAPs), instrumentos para a implementação dos objetivos da Convenção sobre a Diversidade Biológica (CBD, 1992; EPANB, 2017). Os PANs reúnem informações sobre ameaças que recaem 
sobre as espécies, bem como objetivos e ações estratégicas para a mudança no estado de conservação de uma espécie-alvo (e.g. ICMBio, 2020). Até o momento, 192 países desenvolveram NBSAPs e milhares de espécies são cobertas por centenas de PANs globalmente (CBD NBSAPs, 2020). No Brasil, os PANs são responsabilidade institucional do Instituto Chico Mendes de Conservação da Biodiversidade (ICMBio), e 71 PANs já foram executados ou estão em execução no país (ICMBio, 2020).

Instrumentos como os PANs servem para reunir e priorizar estratégias e metas, facilitando a tomada de decisões para o enfrentamento do risco de extinção experimentado por vários alvos de conservação. Estes documentos são instrumentos de gestão, com a premissa de serem construídos de forma participativa, e que deverão ser utilizados para o ordenamento das ações para a conservação de seres vivos e ambientes naturais, levando em consideração um objetivo definido e uma escala temporal (Brasil, 2018). Neste sentido, priorização é um dos pontos críticos de um PAN, pois as ações neles contidas precisam ser classificadas quanto ao nível de prioridade, sendo apresentadas em ordem de importância, relevância e urgência para o alcance dos objetivos estabelecidos, como tem-se observado em diversos NBSAPs elaborados em diferentes países (CBD NBSAPs, 2020). No Brasil, o processo de elaboração dos planos de ação pelo ICMBio é baseado no documento Strategic Planning for Species Conservation: a Handbook (IUCN, 2008), e respaldado pela Instrução Normativa número 21 do ICMBio, de 18/12/2018 (Brasil, 2018). O documento da IUCN prevê a possibilidade de priorização de ações, entretanto não aponta esta priorização como obrigatória. Porém, um argumento pró-priorização é que sem este tipo de indicação de prioridade os agentes executores dessas ações poderiam experimentar dificuldades para escolher quais delas deveriam ser realizadas com mais urgência, ou quais deveriam receber os recursos eventualmente disponibilizados para a execução dos PANs. Assim, a indicação clara de uma ordem de prioridades deve ser vista como positiva e de acordo com as melhores práticas de gestão (e.g. Sipahi \& Timor, 2010; Mukherjee et al., 2018; Zabala et al., 2018), incluindo aquelas voltadas especificamente para a conservação da biodiversidade (e.g., Chow \& Sadler, 2010; Game et al. 2013; Cress et al., 2016).
Apesar da existência de centenas de PANs globalmente, poucos são os instrumentos disponíveis para a avaliação da sua eficiência (e.g., www.trackingactionplans.org) e - até onde vai nosso conhecimento - nenhuma iniciativa no Brasil se propôs a analisar se as ações propostas por estes planos são ou não consensuais. Neste estudo, realizamos um exercício de priorização e busca de consenso para a conservação da biodiversidade usando a AHP para ranquear os objetivos propostos por três PANs em vigência no Brasil. Este ranqueamento permitiu 1) comparar se a ordenação das ações nestes PANs corresponde às ações consideradas prioritárias pelo público que elaborou este documento, 2) identificar possíveis idiossincrasias entre o que diz um documento oficial de política pública e o que pensam as pessoas responsáveis pela execução dessas ações, e 3) identificar ações prioritárias comuns a mais de um PAN - e que podem otimizar o processo de conservação da biodiversidade. Ao analisar a opinião de especialistas e utilizar ferramentas objetivas de ranqueamento de prioridades, este foi um exercício pioneiro para a busca de consenso na conservação da biodiversidade do Brasil, um país megadiverso e que experimenta fortes pressões $e$ ameaças à sua biodiversidade. A metodologia aqui utilizada, bem como os resultados obtidos, pode servir de exemplo para análises similares em outros países ou análises focadas em grupos biológicos específicos.

\section{Material e Métodos}

Escolha dos PANs - Alguns países megadiversos incorporaram os PANs em suas políticas públicas de conservação da biodiversidade bem antes da publicação da Convenção da Diversidade Biológica, em 1992. Este é o caso do Brasil, cujos instrumentos legais que suportam ações para a conservação de espécies datam do começo da década de 1980 (Brasil, 1981; Brasil 2002; EPANB, 2016). No Brasil, os PANs são responsabilidade institucional do Ministério do Meio Ambiente (Brasil, 2003). Embora legalmente reconhecidos desde 1981, mais de 20 anos se passaram até a publicação do primeiro PAN no Brasil, focado no mutum-do-sudeste (Crax blumenbachii), publicado apenas em 2004 (IBAMA, 2004). Desde 2011 a elaboração e implementação dos PANs são responsabilidade do Instituto Chico Mendes de Conservação da 
Biodiversidade (ICMBio). Atualmente 71 PANs foram ou estão em execução no Brasil, cobrindo 884 espécies (ICMBio, 2020). Em consulta ao site do ICMBio (http://www.icmbio.gov.br/portal/ faunabrasileira/planos-de-acao-nacional) foram identificados 46 PANs que estavam em execução até o início de 2016. Destes, foram selecionados apenas os PANs que tinham não mais que três anos desde a sua publicação, e que possuíam uma matriz de planejamento definida, na qual constavam os objetivos que o plano pretende alcançar durante o tempo de sua execução. Os escolhidos foram:

- O PAN para Conservação da Herpetofauna Ameaçada da Mata Atlântica Nordestina (ICMBio, 2013), daqui em diante PANHN, cujo objetivo geral é "aumentar o conhecimento sobre as espécies-foco e minimizar o efeito das ações antrópicas de forma a contribuir para a conservação das espécies de anfíbios e répteis contempladas no PAN da Mata Atlântica nordestina, em cinco anos". Este PAN contém cinco objetivos específicos, cada um com 3 a 18 ações, totalizando 45 ações (Apêndice 1).

- O PAN para a Conservação de Pequenos Felinos (ICMBio, 2014), daqui em diante PANPF, cujo objetivo geral é "reduzir a vulnerabilidade de pequenos felinos nos diferentes biomas por meio de ampliação do conhecimento aplicado à conservação, da proteção de habitat, da minimização de conflitos com atividades antrópicas e de ações políticas efetivas, em cinco anos". Ele conta com sete objetivos específicos, cada um com 3 a 12 ações, somando 43 ações (Apêndice 1).

- O PAN para a Conservação do Tatubola (Tolypeutes tricinctus e Tolypeutes matacus; ICMBio, 2014), daqui em diante PANTB, cujo objetivo é a "redução do risco de extinção do Tolypeutes tricinctus para a categoria Vulnerável e a avaliação adequada do estado de conservação do Tolypeutes matacus". Este plano tem seis objetivos específicos, com 3 a 10 ações cada, resultando em 38 ações (Apêndice 1).

As regras para a elaboração dos PANs não são claras quanto a apresentação das ações em relação à sua importância, relevância e urgência. Os PANs publicados até 2008 contavam com uma classificação de seus objetivos em uma escala de prioridade (baixa, média, alta, essencial ou fundamental), além de indicação de escala de prazos, o que é previsto no documento norteador da IUCN (IUCN, 2008). Porém, esta prática parece ter sido abandonada pelo ICMBio nos PANs a partir de 2009, e não há um consenso se estes PANs apresentam suas ações em ordem de importância durante sua publicação. Em contato pessoal com três analistas do ICMBio envolvidos com a elaboração de PANs, as respostas sobre a necessidade de priorização não foram consensuais, mas houve indicação que nos novos planos a ordem de aparecimento das ações indicava o ranking de prioridade. Então, adotamos essa informação como premissa para podermos comparar a ordem identificada pelos entrevistados com aquela que aparecia nos planos.

Elaboração da AHP - A aplicação da AHP tem sido feita em diversas áreas, principalmente na forma de gestão de operações, como nos setores de produção, energia, transporte, construção civil, marketing, saúde, e crescentemente nas áreas de gestão ambiental e agricultura (Sipahi \& Timor, 2010; Subramanian \& Ramanathan, 2012). Estudos incluem, por exemplo, manejo de recursos pesqueiros (Rossetto et al., 2015), manejo de recursos hídricos (Yavuz \& Baycan, 2013), prioridades para recuperação de minas abandonadas (Kubit et al., 2015) e preferências para preservação de terras (Duke \& Aull-Hyde, 2002). A AHP se baseia em comparações pareadas de alternativas (critérios) para o alcance de um objetivo geral (Saaty, 1990) e os critérios a serem analisados devem: a) Representar bem o problema, da maneira mais completa, porém sem perder a sensibilidade à mudança nos elementos; b) Considerar o ambiente que o problema envolve; e c) Identificar contribuições para a solução do problema e os participantes relacionados ao problema. Aqui, o objetivo geral definido em cada um dos PANs foi apresentado como o objetivo a ser alcançado por quem responderia a AHP, $e$ os objetivos específicos passaram a representar os critérios a serem julgados com relação à sua importância para o alcance do objetivo geral.

O modelo utilizado para a aplicação da AHP foi a planilha eletrônica em Excel proposta por Goepel (2015). Nela os critérios a serem julgados são apresentados aos pares ao participante, que deve escolher qual dos dois é o mais importante e 
quão mais importante ele é, seguindo uma escala de 1 a 9. Ao final do exercício, o participante terá comparado cada uma das opções possíveis de pareamento e o resultado indicará quais critérios são considerados prioritários em relação aos demais, por meio da geração de uma matriz recíproca. Em cada matriz recíproca gerada para as comparações pareadas de cada participante, os dados foram analisados usando a técnica do valor próprio, ou eigenvalue. Também, de acordo com as propriedades das matrizes recíprocas, foi possível calcular a razão de consistência $(\mathrm{CR})$, que é aceitável quando menor ou igual a $10 \%$ (Ananda \& Herath, 2003). A geração de uma matriz consolidada com os resultados de vários participantes permite a obtenção de um ranking geral para as alternativas, permitindo a classificação daquelas vistas como mais ou menos prioritárias.

\section{Consulta aos especialistas e coleta de dados}

Foram escolhidos para a aplicação da AHP e construção dos rankings especialistas que colaboraram com a elaboração de cada um dos PANs. Assim, foi solicitada às Coordenações desses PANs uma lista de nomes e contatos dos participantes. Essa lista foi complementada com os nomes de membros das instituições comprometidas com a realização de cada um dos PANs, de acordo com suas portarias de assessoramento técnico. As pessoas foram contatadas e convidadas a participarem da pesquisa aqui descrita.

Os participantes dos PANs representavam diferentes instituições localizadas em diferentes cidades do Brasil. Assim, o contato foi feito via e-mail e telefone, entre maio de 2015 e setembro de 2016. Para cada participante foi enviada a planilha AHP referente ao seu respectivo PANs, $e$ instruções sobre o seu preenchimento. $\mathrm{O}$ mesmo arquivo ainda continha um Termo de Consentimento Esclarecido explicando riscos e benefícios da pesquisa, para que o participante pudesse ler e assinalar se concordava ou não participar. As listas utilizadas para contatar os participantes continham de 27 a 67 pessoas (Apêndice 1). A lista de participantes do PANTB foi obtida por meio de pesquisa e contato com as instituições que constavam na portaria que estabeleceu o Grupo de Assessoramento Técnico desse PAN (Brasil, 2014b). O PANHN incluía especialistas nos grupos e representantes de sete dos nove estados nordestinos, incluindo instituições como as principais universidades $e$ institutos de pesquisa desses estados, Polícia Militar e órgãos governamentais estaduais $e$ municipais, como secretarias de meio ambiente, além de funcionários do ICMBio (Tabela 1). O PANPF contava com 25 instituições diferentes entre especialistas nos táxons, de instituições de ensino e pesquisa (e.g. UFLA, UEMA, UFSCAR, UFMG, USP), representantes de Unidades de Conservação (e.g. Parna Iguaçu, Parna Serra dos Órgãos, Parna Serra da Capivara, Esec Taimã), Sociedades de Zoológicos, Ibama, consultorias e secretarias de meio ambiente. Para o PANTB, foram contatados membros do ICMBio, Associação Caatinga, Unidades de Conservação (Reserva Serra das Almas, ESEC Raso da Catarina), Embrapa, instituições de Pesquisa (UFPI, UFPB, USP) e outros órgãos. Dos 112 participantes identificados nos três PANs, 99 foram contatados e 28 aceitaram participar da pesquisa. As participações por PAN variaram entre $18 \%$ (PANHN) e $52 \%$ (PANTB), incluindo pessoas de diferentes instituições e perfis, mas que participaram da elaboração desses planos (Apêndice 1). A pesquisa foi autorizada pelo Comitê de Ética em Pesquisa Envolvendo Seres Humanos da Universidade Federal de Pernambuco, com parecer de número 1.392.293.

\section{Análise dos dados}

A execução da AHP permitiu gerar um ranking consolidado de objetivos identificados como prioritários para cada um dos PANs, baseado nos pesos finais obtidos por cada opção. PANs diferentes podiam apresentar objetivos específicos semelhantes (p. ex., "levantamento de informações básicas", ou "recuperação de habitat"). Esses casos possibilitaram uma análise comparativa da classificação desses objetivos semelhantes nos diferentes rankings AHP gerados.

Para identificar objetivos prioritários comuns a mais de um PAN foi utilizado o PANTB, único dos três cujas ações e os objetivos específicos já se apresentavam em uma ordem de importância em suas matrizes de planejamento, definidas durante as oficinas desse PAN (M.M.V. Montenegro, com. pes.). Isto permitiu a comparação dessa ordem pré-definida com a ordem do ranking gerado através da AHP. 


\section{Resultados}

\section{Rankings dos PANs $\times$ AHP}

Os rankings gerados por meio da AHP resultaram em uma ordenação de prioridades que representa a opinião dos participantes sobre os objetivos específicos de cada PAN, e houve pouca congruência entre a ordenação dos objetivos constantes nos PANs e aquelas obtidas pela AHP: apenas cinco das 18 ações permaneceram nas mesmas posições. Para o PANPF, o ranking obtido com a AHP foi totalmente diferente daquele listado no PAN, com uma total inversão de prioridades (Tabela 1).

Tabela 1 - Objetivos específicos dos planos nacionais para a conservação da herpetofauna do Nordeste, pequenos felinos e tatu-bola, e seus respectivos pesos atribuídos através da análise hierárquica de processos aplicada aos participantes de cada plano. Para fins de comparação entre os planos, as ações foram sintetizadas em temas comuns, apresentados em negrito e em parênteses. Baseado nos pesos de cada objetivo obtém-se um ranking de prioridades (Rk) e é possível comparar este ranking com a ordem em que cada objetivo específico é apresentado em cada plano. As setas representam se a ordem de prioridade entre a AHP e o PAN se manteve (setas horizontais), se houve redução de prioridade (seta para baixo), ou aumento de prioridade (seta para cima).

\section{PAN Herpetofauna do Nordeste - Objetivos específicos}

1 Promover a manutenção, a ampliação e restabelecer a conectividade das áreas que incluem os habita das espécies contempladas no PAN (conectividade de habitat)

2 Ampliar o conhecimento sobre a história natural, biogeografia e sistemática das espécies contempladas no PAN (conhecimento básico)

3 Promover a mudança na percepção das populações humanas sobre a importância biológica de répteis e anfíbios nas áreas estratégicas do PAN (educação ambiental)

4 Ampliar as parcerias entre órgãos públicos, setor produtivo e sociedade civil organizada (parcerias estratégicas)

5 Reduzir os impactos negativos às espécies contempladas no PAN causados pelo manejo inadequado dos recursos naturais (redução de impactos)

\section{PAN Pequenos Felinos - Objetivos específicos}

1 Compreender como a caça e o abate por retaliação afetam as diferentes populações de pequenos felinos em cada bioma, em cinco anos (impacto da caça/perseguição)

2 Reduzir a remoção ilegal (caça, abate, retirada de animais vivos) de indivíduos das diferentes populações de pequenos felinos, em cinco anos (redução de mortes)

3 Dimensionar e minimizar os impactos da co-ocorrência entre pequenos felinos e animais domésticos e exóticos, em cinco anos (co-ocorrência)

4 Ampliar o conhecimento sobre os impactos das doenças na saúde das populações de pequenos felinos, em cinco anos (impacto de doenças)

Manter e ampliar o conhecimento sobre os impactos das doenças na saúde das

5 populações de pequenos felinos e reduzir os processos de fragmentação e perda de habitat nas suas áreas de ocorrência, considerando os impactos nas diversas escalas, em cinco anos (conectividade de habitat)

Estimular a criação e implementação de políticas públicas que determinem a

6 redução da fragmentação e perda de habitat em toda área de distribuição de pequenos felinos, em cinco anos (redução da perda de habitat)

Realizar estudos que avaliem de que forma os diferentes processos naturais e

7 antrópicos influenciam a conservação das populações de pequenos felinos, em cinco anos (conhecimento básico)

\begin{tabular}{|c|c|c|}
\hline Pesos & Rk & PAN/AHP \\
\hline $39,6 \%$ & 1 & \\
\hline $19,8 \%$ & 2 & \\
\hline $14,6 \%$ & 4 & \\
\hline $10,5 \%$ & 5 & \\
\hline $15,6 \%$ & 3 & \\
\hline Pesos & $\mathbf{R k}$ & PAN/AHP \\
\hline $8,8 \%$ & 5 & \\
\hline $14,5 \%$ & 3 & \\
\hline $7,2 \%$ & 7 & \\
\hline $8,3 \%$ & 6 & \\
\hline $28,3 \%$ & 1 & \\
\hline $20,3 \%$ & 2 & \\
\hline $12,4 \%$ & 4 & \\
\hline
\end{tabular}




\begin{tabular}{c|l|c|}
\multicolumn{1}{c}{ PAN Tatu-bola - Objetivos Específicos } & Pesos \\
\hline 1 & $\begin{array}{l}\text { Atualizar as áreas de ocorrência das espécies (Tolypeutes tricinctus e Tolypeutes } \\
\text { matacus) e avaliar as principais ameaças ao longo de suas distribuições geográficas } \\
\text { (distribuição e ameaças) }\end{array}$ \\
\hline 2 & $\begin{array}{l}\text { Mobilizar as comunidades locais, em áreas de ocorrência de Tolypeutes tricinctus, } \\
\text { bem como a sociedade em geral, sobre a importância da proteção da espécie na } \\
\text { Caatinga e no Cerrado (educação ambiental) }\end{array}$ & PAN/AHP \\
\hline 3 & $\begin{array}{l}\text { Ampliar o conhecimento sobre a biologia e ecologia (dinâmica populacional, } \\
\text { variabilidade genética e vulnerabilidade às alterações antrópicas) para o } \\
\text { direcionamento de estratégias de conservação dos tatus-bola (Tolypeutes tricinctus e } \\
\text { Tolypeutes matacus) (conhecimento básico) }\end{array}$ & $17,6 \%$ \\
\hline 4 & $\begin{array}{l}\text { Ampliar, qualificar e integrar a fiscalização para coibir a caça do tatu-bola } \\
\text { (Tolypeutes tricinctus) (fiscalização contra a caça) }\end{array}$ \\
\hline 5 & $\begin{array}{l}\text { Reduzir a taxa de perda de habitat de Tolypeutes tricinctus nos próximos cinco anos } \\
\text { (redução da perda de habitat) }\end{array}$ & $20,7 \%$ \\
\hline 6 & $\begin{array}{l}\text { Promover a conectividade entre as populações de Tolypeutes tricinctus nos } \\
\text { próximos cinco anos (conectividade de habitat) }\end{array}$ \\
\hline
\end{tabular}

\section{Comparação de objetivos similares}

Quatro ações foram comuns a mais de um PAN: 1) o reestabelecimento da conectividade das populações; 2) a ampliação do conhecimento básico sobre as espécies-alvo; 3) ações de educação ambiental; e 4) redução de impactos e ameaças.

O ranking gerado pela AHP para o PANHN colocou a conectividade de habitat em primeiro lugar $(39,6 \%)$, com quase $10 \%$ de peso a mais que o segundo lugar (Tabela 1 ). A conectividade de habitat (28,3\%) também ficou em primeiro lugar no PANPF, com quase $8 \%$ a mais que o segundo lugar, a redução da perda de habitat (Tabela 1). No PANTB os objetivos relacionados à ampliação da conectividade dos animais (objetivo 6) e recuperação do habitat (objetivo 5) estavam propostos separadamente: a conectividade ficou na última posição do ranking pela AHP, enquanto a diminuição da perda de habitat ficou na segunda posição (Tabela 1).

A ampliação do conhecimento foi identificada como prioritária no PANHN e PANTB (Tabela 1). Já para o PANPF, ficaram nas últimas posições no ranking. Os objetivos relacionados à educação ambiental das comunidades envolvidas com as espécies ficaram na quarta posição nos PANHN e PANTB. Objetivos relacionados à redução de impactos e ameaças ocuparam posições distintas dependendo do PAN analisado (Tabela 1).

\section{Discussão}

Utilizando a análise hierárquica de processos (AHP) foi possível ranquear os objetivos específicos de três planos de ação para a conservação de espécies ameaçadas de extinção (PAN) brasileiras. Os resultados indicam uma falta de congruência entre os objetivos ordenados nos PANs e o ranking obtido pela AHP com os especialistas que elaboraram estes mesmos PANs. Um conjunto de objetivos comuns foi identificado como prioridade pela AHP em mais um PAN (p. ex., recuperação do habitat e conectividade entre populações). Entretanto, nem sempre estes objetivos comuns foram prioritários em seus respectivos PANs. A falta de congruência observada pode comprometer a execução de alguns dos objetivos gerais, prejudicando ou até inviabilizando a conservação das espécies-alvo desses documentos.

Mesmo sendo comumente citados como ferramentas de apoio à decisão, PANs são, antes de qualquer coisa, um esboço de prioridades, identificando e informando aos tomadores de decisão sobre onde alocar recursos, quando 
disponíveis (Game et al., 2013). Uma parte fundamental na elaboração dos PANs é a análise $\mathrm{de}$ ameaças, que direciona o estabelecimento dos seus objetivos e estruturação das suas ações (Brasil, 2018). Apesar disso, a forma como os PANs devem organizar as ações prioritárias dentro de sua metodologia de elaboração e execução não é clara e gera dúvidas até mesmo entre seus analistas. Embora haja uma tentativa de priorização em alguma escala - como no relato de que no PANTB as ações foram construídas e apresentadas em ordem de prioridade - essa prática não parece ser a regra nos demais PANs, e não há instrução direta nos documentos que norteiam sua elaboração (e.g. Instrução Normativa ICMBio $n^{\circ}$ 21, de 18 de dezembro de 2018; Guia para Gestão de Planos de Ação Nacional para a Conservação das Espécies Ameaçadas de Extinção, ICMBio, 2018). Esta falta de clareza e padronização é preocupante, pois, apesar de serem construídos de forma a direcionar ações para neutralizar/reduzir ou mitigar as principais ameaças às espécies-alvo, a falta de indicação clara pode fazer com que um PAN não necessariamente esteja executando aquelas ações que, de fato, devem ser vistas como prioritárias.

Com o foco no combate das ameaças levantadas, os PANs devem abranger em suas ações a interferência em políticas públicas, o desenvolvimento de conhecimentos específicos, a sensibilização de comunidades e o controle da ação humana (ICMBio, 2018). Ações desse tipo são previstas recorrentemente nos objetivos específicos dos três PANs analisados neste estudo (i.e. levantamento de conhecimentos básicos e iniciativas de educação ambiental). $\mathrm{O}$ produto gerado através da AHP permitiu uma análise mais detalhada de cada ameaça, gerando um ranking que é reconhecido pelos entrevistados como aquele que contém ameaças em uma ordem de prioridades. Quando esta ordem de prioridades é clara, o seu enfrentamento pode ser mais eficiente, pode poupar recursos financeiros e tempo (Game et al., 2013). Dessa forma, este tipo de ferramenta pode fortalecer as medidas para a conservação da biodiversidade, aumentando a obtenção de resultados positivos.

A AHP foi bastante útil para indicar a falta de congruência entre a ordem de ações proposta na apresentação dos próprios PANs e a ordem que seus realizadores identificam como realmente prioritária. Dos três planos analisados, apenas o mais recente, o PAN do Tatu-bola, possuía um sumário executivo publicado, no qual constavam as informações levantadas para embasar seus objetivos. No documento, as principais ameaças destacadas para as espécies foram a destruição e alteração de habitat, e a caça (ICMBio, 2014), as quais estavam contempladas nos objetivos propostos pelo PAN (Brasil, 2014b). Os objetivos referentes a essas ameaças (5 e 4, respectivamente) apareciam na quinta e quarta posições, na matriz de planejamento desse PAN, porém, no ranking AHP a ordem de prioridades diferiu daquela proposta pelo PAN. A redução da taxa de perda de habitat de Tolypeutes tricinctus, espécie distribuída majoritariamente na Caatinga e citada no sumário executivo do PNA como uma das espécies de tatu mais sensíveis às alterações do ambiente onde vive (ICMBio, 2014), passou do quinto para o segundo lugar no ranking AHP. Por outro lado, ampliar, qualificar e integrar a fiscalização para coibir a caça do tatu-bola ( $T$. trincinctus) caiu uma posição (da quarta para a quinta). Diferentemente do esperado, promover a conectividade entre as populações de T. trincinctus (objetivo 6), que está intimamente ligado à redução da perda de habitat, permaneceu na última colocação para este plano. O sumário também destaca que as duas espécies-alvo do PAN são as espécies de tatu menos conhecidas do Brasil (ICMBio, 2014), o que se reflete na permanência da priorização do levantamento de informações sobre distribuição, biologia e ecologia das espécies-alvo tanto na proposta original do PAN quanto no ranking gerado através da AHP (objetivos 1 e 3 ).

Os PANs podem frequentemente lidar com um número elevado de espécies-alvo $e$ ações, $e$ isso reforça a necessidade de um ordenamento claro de quais são as prioridades de enfrentamento em relação às ameaças experimentadas por estas espécies (Game et al., 2013; Carwardine et al., 2012). O PANHN é um exemplo: dos três PANs abordados no estudo, ele é o que abrange o maior número de espécies-alvo e espécies beneficiadas, sendo 25 espécies nacionalmente ameaçadas de extinção (8 anfíbios, 2 anfisbênias, 8 lagartos e 7 serpentes), nove quase ameaçadas (5 anfíbios, 3 anfisbêniase 1 lagarto) e 39 com dados insuficientes (28 anfíbios, 2 anfisbênias, 3 lagartos, 1 quelônio e 5 serpentes; Brasil, 2016). A AHP ressaltou que, para este PAN, a ampliação do conhecimento básico sobre tais espécies é a prioridade 1. Isso pode ser devido ao elevado número de espécies 
abrangidas, principalmente aquelas deficientes de dados. Ainda, tal ampliação do conhecimento também é definida como objetivo geral desse PAN (ICMBio, 2013).

Por lidarem com espécies ou grupo diferentes, e que podem estar experimentando pressões e ameaças diferentes, as prioridades entre grupos nem sempre se equivalem, reforçando a necessidade de que, para cada PAN, as ações devem estar bem discriminadas $e$ definidas quanto à ordem de prioridade. O PANPF é aqui um bom exemplo: apesar de incluir a ampliação do conhecimento sobre as espécies como parte do objetivo geral (Brasil, 2014a), a AHP executada apontou que os objetivos específicos relacionados à ampliação do conhecimento sobre as espécies (objetivos 1 e 4) ficaram posicionados entre as últimas colocações no ranking AHP. Este resultado poderia ser atribuído a duas alternativas: 1) o menor número de espécies abrangidas por este plano, sendo quatro ameaçadas nacionalmente de extinção (Leopardus pardalis, Leopardus trigrinus, Leopardus colocolo e Leopardus wiedii) e outras duas beneficiadas que também ocorrem em território nacional (Leopardus geoffroyi e Puma yagouaroundi) (Brasil, 2014a), o que pode ser um fator para tornar mais simples o levantamento de informações; ou 2) a urgência para realização dos outros objetivos propostos devido às ameaças enfrentadas pelo grupo. Este caso ficou refletido na prioridade dada à manutenção e ampliação da conectividade entre populações e redução da fragmentação e perda de habitat (objetivo específico 5), ao estímulo para a implementação de políticas públicas também voltadas para a redução da perda e fragmentação de habitat (objetivo específico 6), e à redução da remoção ilegal de indivíduos das populações (objetivo 2).

\section{Identificação de ações comuns}

A AHP se mostrou especialmente eficaz na identificação de um conjunto de ações que se destacam como prioritárias em mais de um PAN. Os PANs analisados neste estudo abrangem juntos todos os grandes biomas brasileiros: Amazônia, Caatinga, Cerrado, Mata Atlântica, Pampa, Pantanal. As áreas de ocorrência das espécies beneficiadas pelos PANs incluem um ou mais desses biomas e sofrem com a perda de habitat, principalmente na Mata Atlântica $e$ no Cerrado. Entre as consequências dessa perda e fragmentação de habitat está a diminuição de conectividade entre as populações, o que pode levá-las à redução de sua variabilidade e a um consequente declínio no número de indivíduos (Saunders et al., 1991; Haddad et al., 2015). Em consonância com este contexto, os participantes desta pesquisa evidenciaram a importância da manutenção de habitat e da conectividade entre remanescentes para a conservação das espéciesalvo dos PANs. Estes foram os casos do objetivo específico 1 do PANHN e do objetivo específico 5 do PANPF, ambos em primeiro lugar nos respectivos rankings AHP, e do objetivo específico 5 do PANTB, relacionado à diminuição da perda de habitat, que ficou em segundo lugar no ranking AHP.

A preocupação com a perda e fragmentação de habitat se reflete não só no meio acadêmico científico, mas também na prática, através dos instrumentos de tomada de decisão. Evidenciar a importância desse fator através da priorização de ações dentro de tais instrumentos é fundamental para que a prática reflita a percepção que os próprios especialistas envolvidos têm sobre isso. Por meio deste estudo também fica clara a importância de aumentar os esforços para a ampliação de conhecimentos sobre as espécies ameaçadas no Brasil. Também se torna evidente que os planos de ação nacional podem ser mais bem direcionados e eficazes se suas ações consideradas mais relevantes forem priorizadas desde o seu planejamento.

\section{A busca por consenso}

A existência de um consenso entre as partes interessadas quanto às prioridades do planejamento é de fundamental importância para a efetividade do processo (Ban et al., 2009; Crees et al., 2015). Em estudo relacionado às prioridades na conservação de uma área de floresta em Michigan, EUA, Chow e Sadler (2010) investigaram a existência de consenso entre as partes interessadas locais e especialistas externos utilizando AHP associada a outro método, e encontraram divergência entre as prioridades selecionadas para cada um dos grupos. Técnicas como a AHP são úteis para apontar esses tipos $\mathrm{de}$ falhas relacionadas a consenso, tornando o processo de tomada de decisões mais coeso.

Ao escolher as prioridades deve-se destacar a importância de deixar claro o problema que 
se quer resolver, assim como escolher as ações prioritárias que podem ser usadas para a resolução de tal problema, e não apenas os locais, habitat ou espécies relacionadas (Game et al., 2013). No caso dos PANs, o objetivo geral compreende este problema alvo e os objetivos específicos devem ser julgados visando o alcance dele, o que torna estas ferramentas uma peça chave para a conservação. Porém, a priorização das ações dos PANs não tem sido feita de maneira clara, principalmente desde que a prática dos quadros de escala de prioridades foi abandonada, desde 2009. Além disso, a metodologia de elaboração desses PANs também não prevê uma etapa de ordenação de prioridades de forma padronizada (ICMBio, 2012). À vista disso, seria essencial para tornar mais eficaz a conservação das espécies alvo desse documento que tais práticas fossem retomadas e aprimoradas com ferramentas como a AHP utilizada no presente estudo, produzindo rankings consensuais e claros dos objetivos de cada PAN. Recomendamos, desta forma, que a indicação clara de uma escala de prioridade para os objetivos e ações (p. ex., baixa, média, alta, essencial ou fundamental) seja novamente adotada na elaboração de PANs futuros, o que é previsto no documento norteador da IUCN (IUCN, 2008).

A investigação e a implementação de técnicas que ajudem na escolha consensual de prioridades dentro de documentos como os PANs também é de extrema importância para a efetividade de sua aplicação, principalmente do ponto de vista financeiro. A base orçamentária para os planos de ação, administrada pelo ICMBio, provê, dentre outras fontes, do Orçamento Geral da União (Decreto $n^{\circ}$ 7.515/11). Entretanto, o Ministério do Meio Ambiente, que provém os recursos do ICMBio, tem experimentado sucessivos cortes orçamentários (Juras, 2011; Bragança, 2013; Pegurier, 2015), tornando os recursos incertos e reduzidos. Dessa forma, há uma competição por financiamento, já bastante escasso, com outros setores da sociedade, o que caracteriza uma barreira para as iniciativas conservacionistas (Gregory \& Long, 2009). Complementarmente, apesar da suscetibilidade política ao cumprimento das propostas de planos de ação, ao mesmo tempo há um forte apoio do governo a setores políticos cujas áreas de foco são frequentemente contrárias aos objetivos favoráveis à biodiversidade (Sarkki et al., 2015). Em estudo sobre como as National Biodiversity
Strategies and Action Plans contribuem para integrar a biodiversidade em todos os setores políticos na Finlândia, Sarkki et al. (2015) levanta a discussão sobre este trade-off entre a administração ambiental e os setores econômicos, destacando uma lacuna de responsabilidade na política de biodiversidade. Assim, quanto mais claras e organizadas as ações propostas num plano e suas respectivas execuções, maior sua força para competir por recursos. Como demonstrado aqui, a AHP mostrou-se aproveitável para este propósito, tornando mais clara a finalidade dos recursos por apontar os objetivos mais emergenciais para execução.

\section{Perspectivas de uso das análises multicritérios}

Esta pesquisa mostrou que há espaço para a melhoria dos PANs. Estes documentos são bastante proficientes na identificação das pressões e ameaças experimentadas por alvos conservacionistas, e na proposição de ações para neutralizá-las. Ajustes em sua metodologia de elaboração, principalmente no que diz respeito à escolha consensual de objetivos $e$ ações prioritárias, tornarão estas ferramentas mais sólidas e seus resultados cada vez mais positivos. O uso de metodologias que facilitem a implementação dessa priorização nos planos de ação - tais como a AHP - e em outras ferramentas de gestão para a conservação pode permitir uma expansão desse tipo de organização estratégica.

Se uma AHP ou outra análise multicritério tivesse sido realizada no encerramento da oficina que deu origem a cada um desses PANs, algumas das incongruências apresentadas teriam sido imediatamente sanadas, e os PANs já seriam publicados em sua versão final com cada umas das ações em sua ordem de prioridade. Isso tornaria mais evidente quais são, de fato, as ações que os especialistas consultados para a elaboração do PAN realmente reconhecem como mais importantes. A AHP não é a única e nem a melhor das ferramentas para esse propósito (e.g. ANP, DEA e Delphi - Saaty, 1996; Govindan et al., 2015; Driscoll et al., 2016; Mukherjee et al., 2018; Zabala et al., 2018), mas sua simplicidade e objetividade as colocam como uma candidata. Sugere-se o teste de outros métodos e a aplicação deles durante a elaboração dos PANs, sendo também inseridos oficialmente nos seus mecanismos de 
elaboração, para que sejam colocados em prática seguindo a ordem de relevância de seus objetivos para o alcance de um objetivo maior. De qualquer forma, o objetivo final, ou seja, a conservação da biodiversidade, precisa ser visto de maneira ampla e modificações nas formas tradicionais podem ser benéficas desde que busquem sanar problemas como o da falta de consenso ou das falhas de planejamento estratégico.

\section{Agradecimentos}

Este artigo é parte da Dissertação de Mestrado de E.C.A. Souza junto ao Programa de Pós Graduação em Biologia Animal da Universidade Federal de Pernambuco (PPGBA/UFPE), $e$ agradecemos aos Drs. C. B. Valladares-Padua, R.J. Ladle e P. M. Sales por seus comentários construtivos. Agradecemos ao PPGBA/UFPE e à Fundação de Amparo à Ciência e Tecnologia de Pernambuco (FACEPE) pela concessão da bolsa de Mestrado. Agradecemos aos participantes e coordenações de Planos de Ação Nacionais, que voluntariamente participaram da pesquisa, e sem os quais o trabalho não teria sido realizado. E. Bernard é bolsista de produtividade do $\mathrm{CNPq}$.

\section{Referências}

Ananda J \& Herath G. The use of Analytic Hierarchy Process to incorporate stakeholder preferences into regional forest planning. Forest Policy and Economics, 5(1): 13-26, 2003.

Ban NC, Picard CR \& Vincent AC. Comparing and integrating community-based and science-based approaches to prioritizing marine areas for protection. Conservation Biology, 23(4): 899-910, 2009.

Bragança D. "O passivo fundiário é só a ponta do iceberg", afirma Vizentin. O Eco, 03 set. 2013. Disponível em: <http://www.oeco.org.br/ reportagens/27548-o-passivo-fundiario-e-so-aponta-do-iceberg-afirma-vizentin/>. Acesso em: 14/12/2017.

Brasil. 2013. Portaria MMA/ICMBio $n^{\circ} 200$, de $1^{\circ}$ de Julho de 2013. Diário Oficial da União. $<$ https://www.icmbio.gov.br/cepsul/images/stories/ legislacao/Portaria/2016/p icmbio_200_2013_pan herpetofauna_mataat $\%$ C $3 \%$ A2ntica_nordestina. pdf $>$. Acesso em: 14/12/2020.

Brasil. 2014. Portaria MMA/ICMBio $n^{\circ} 32,27$ de Março de 2014a. Diário Oficial da União. < https:// www.icmbio.gov.br/portal/images/stories/docs-pan/ pan-pequenos-felinos/1-ciclo/pan-pequenos-felinosportaria-aprovacao.pdf $>$. Acesso em: 14/12/2020.

Brasil. 2014. Portaria MMA/ICMBio $\mathrm{n}^{\circ} 56,22$ de Maio de 2014b. Diário Oficial da União. < https:// www.icmbio.gov.br/portal/images/stories/o-que-somos/ Port_56.pdf>. Acesso em: 14/12/2020.

Brasil. 1981. Lei no 6.938, de 31 de agosto de 1981. Diário Oficial da União. <http://www.planalto.gov.br/ ccivil_03/leis/16938.htm > . Acesso em: 14/12/2020.

Brasil. 2016. Portaria ICMBio $\mathrm{n}^{\circ} 38$, de 03 de maio de 2016. Diário Oficial da União. < https://ava.icmbio.gov. br/cepsul/images/stories/legislacao/Portaria/2016/p_ icmbio_38_2016_pan_herpetofauna_ne.pdf $>$. Acesso

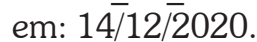

CBD NBSAPs. National Biodiversity Strategies and Action Plans (NBSAPs). Disponível em: <https://www. cbd.int/nbsap/>. Acesso em 06/08/2020.

Chow TE \& Sadler R. The consensus of local stakeholders and outside experts in suitability modeling for future camp development. Landscape and Urban Planning, 94(1): 9-19. 2010.

Cress JJ et al. A comparative approach to assess drivers of success in mammalian conservation recovery programs. Conservation Biology, 30(4): 694-705. 2016.

Driscoll DA, Bode M, Bradstock RA, Keith DA, Penman TD \& Price OF. Resolving future fire management conflicts using multicriteria decision making. Conservation Biology, 30(1): 196-205. 2016.

Duke JM \& Aull-Hyde R. Identifying public preferences for land preservation using the analytic hierarchy process. Ecological Economics, 42(1): 131-145. 2002.

EPANB - Estratégia e Plano de Ação Nacionais para a Biodiversidade 2016-2020. Ministério do Meio Ambiente - MMA, Secretaria de Biodiversidade e Florestas - SBF. 2017. Disponivel em: < https://www. mma.gov.br/images/arquivo/80049/EPANB/EPANB_ PORT.pdf> Acesso em: 06/08/2020

Game ET, Kareiva P \& Possingham HP. Six common mistakes in conservation priority setting. Conservation Biology, 27(3): 480-485. 2013.

Goepel K. AHP Excel Template Version. < http://bpmsg. com/category/ahp/>. Acesso em: 06/08/2020.

Govindan K, Diabat A \& Shankar KM. Analyzing the drivers of green manufacturing with fuzzy approach. Journal of Cleaner Production, 96: 182-193. 2015.

Gregory R, Long G, Colligan M, Geiger JG \& Laser $M$. When experts disagree (and better science won't help much): using structured deliberations to support endangered species recovery planning. Journal of Environmental Management, 105: 30-43. 2012. 
Gregory R \& Long G. Using structured decision making to help implement a precautionary approach to endangered species management. Risk Analysis, 29(4): 518-532. 2009.

Haddad NM et al. Habitat fragmentation and its lasting impact on Earth's ecosystems. Science Advances, 1(2): e1500052. 2015.

IBAMA. Plano de Ação para a Conservação do Mutum-do-sudeste Crax blumenbachii. Série Espécies Ameaçadas, Volume 1. 2004 < http://www. icmbio.gov.br/portal/faunabrasileira/plano-de-acaonacional-lista/2730-plano-de-acao-nacional-para-aconservacao-do-mutum-do-sudeste $>$. Acesso em: 06/08/2020.

Instituto Chico Mendes de Conservação da Biodiversidade (ICMBio). 2018. Guia para Gestão de Planos de Ação Nacional para a Conservação das Espécies Ameaçadas de Extinção - PAN ELABORE - MONITORE - AVALIE. ICMBio. Brasília: ICMBio. 160p.

Instituto Chico Mendes de Conservação da Biodiversidade (ICMBio). 2014. Sumário Executivo do Plano de Ação Nacional para a Conservação do Tatu-bola. <http://www.icmbio.gov.br/portal/faunabrasileira/ plano-de-acao-nacional-lista/4808-plano-de-acaonacional-para-conservacao-do-tatu-bola $>$. Acesso em: 06/08/2020.

Instituto Chico Mendes de Conservação da Biodiversidade (ICMBio). 2020. Planos de Ação Nacional. <http://www.icmbio.gov.br/portal/faunabrasileira/ planos-de-acao-nacional>. Acesso em: 06/08/2020.

Brasil. 2018. Instrução Normativa MMA/ICMBio n 21, de 18 de dezembro de 2018. Diário Oficial da União. $<$ https://www.icmbio.gov.br/portal/images/stories/docsplano-de-acao-ARQUIVO/00-saiba-mais/01_-_IN ICMBIO_N\%C2\%BA_21_DE_18_DE_DEZ_DE_- 2018. pdf>. Ačesso em: 14/12/2020.

IUCN/SSC. 2008. Strategic Planning for Species Conservation: A Handbook. Version 1.0. Gland, Switzerland: IUCN Species Survival Commission. 104p.

Jacobs MH, Vaske JJ \& Sijtsma MT. Predictive potential of wildlife value orientations for acceptability of management interventions. Journal for Nature Conservation, 22(4): 377-383, 2014.

Juras IAGM. Breves comentários sobre a base constitucional da proteção da biodiversidade. in Conservação da biodiversidade: legislação e políticas públicas. Brasília: Câmara dos Deputados, 131, 2011.
Kubit OE, Pluhar C \& de Graff JV. A model for prioritizing sites and reclamation methods at abandoned mines. Environmental Earth Sciences, 73(12): 7915-7931. 2015.

McShane TO et al. Hard choices: making trade-offs between biodiversity conservation and human wellbeing. Biological Conservation, 144(3): 966-972. 2011.

Mukherjee N, Zabala A, Huge J, Nyumba TO, Esmail BA \& Sutherland WJ. Comparison of techniques for eliciting views and judgements in decision-making. Methods in Ecology and Evolution, 9(1): 54-63. 2018.

Pegurier E. 2015. Angela Kuczach: "Precisamos das UCs para existir". O Eco, 03 fev. 2015. Disponível em: <http://www.oeco.org.br/reportagens/28904-angelakuczach-precisamos-das-ucs-para-existir/> . Acesso em: 06/08/2020.

Redpath SM et al. Understanding and managing conservation conflicts. Trends in Ecology \& Evolution, 28(2): 100-109. 2013.

Rossetto $\mathrm{M}$ et al. Multi-criteria decision-making for fisheries management: A case study of Mediterranean demersal fisheries. Marine Policy, 53: 83-93. 2015.

Saaty TL. How to make a decision: the analytic hierarchy process. European journal of operational research, 48(1): 9-26. 1990.

Sarkii $S$ et al. Are national biodiversity strategies and action plans appropriate for building responsibilities for mainstreaming biodiversity across policy sectors? The case of Finland. Journal of Environmental Planning and Management, 59(8): 1377-1396. 2015.

Saunders DA, Hobbs RJ \& Margules CR. Biological consequences of ecosystem fragmentation: a review. Conservation biology, 5(1): 18-32. 1991.

Sipahi S \& Timor M. The analytic hierarchy process and analytic network process: an overview of applications. Management Decision, 48(5): 775-808. 2010.

Subramanian N \& Ramanathan R. A review of applications of Analytic Hierarchy Process in operations management. International Journal of Production Economics, 138(2): 215-241. 2012.

Zabala A, Sandbrook C \& Mukherjee N. When and how to use $\mathrm{Q}$ methodology to understand perspectives in conservation research. Conservation Biology, 32(5): 1185-1194. 2018.

Yavuz F \& Baycan T. Use of swot and analytic hierarchy process integration as a participatory decision making tool in watershed management. Procedia Technology, 8: 134-143. 2013. 


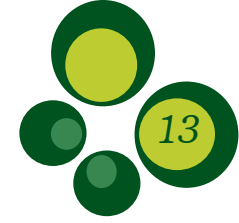

Apêndice 1 - Detalhamento dos planos de ação nacional para a Conservação (PANs) utilizados em um exercício para busca de consenso em conservação da biodiversidade utilizando análise hierárquica de processos (AHP): ano de publicação, objetivos, número de ações, número de participantes de sua elaboração $e$ parcela que aceitou realizar a AHP para o ranqueamento dos objetivos específicos de cada PAN de acordo com sua prioridade.

\begin{tabular}{|c|c|c|c|c|c|c|c|}
\hline PAN (ano) & Objetivo geral & & Objetivos específicos & Ações & $\begin{array}{l}\text { Total } \\
\text { de } \\
\text { ações }\end{array}$ & $\begin{array}{l}\text { Especialistas } \\
\text { no PAN/ } \\
\text { especialistas } \\
\text { contatados }\end{array}$ & $\begin{array}{c}\text { Aceites } \\
\text { para a } \\
\text { pesquisa } \\
(\%)\end{array}$ \\
\hline \multirow{5}{*}{$\begin{array}{l}\text { PAN } \\
\text { Herpetofauna } \\
\text { do Nordeste } \\
\text { (2013) }\end{array}$} & \multirow{5}{*}{$\begin{array}{l}\text { Aumentar o } \\
\text { conhecimento } \\
\text { sobre as espécies- } \\
\text { foco e minimizar } \\
\text { o efeito das ações } \\
\text { antrópicas de forma } \\
\text { a contribuir para } \\
\text { a conservação } \\
\text { das espécies de } \\
\text { anfíbios e répteis } \\
\text { contempladas } \\
\text { no PAN da } \\
\text { Mata Atlântica } \\
\text { nordestina, em } \\
\text { cinco anos }\end{array}$} & 1 & $\begin{array}{l}\text { Promover a manutenção, a ampliação e } \\
\text { restabelecer a conectividade das áreas que } \\
\text { incluem os habitat das espécies contempladas } \\
\text { no PAN }\end{array}$ & 18 & \multirow{5}{*}{45} & \multirow{5}{*}{$67 / 50$} & \multirow{5}{*}{$9(18 \%)$} \\
\hline & & 2 & $\begin{array}{l}\text { Ampliar o conhecimento sobre a história } \\
\text { natural, biogeografia e sistemática das } \\
\text { espécies contempladas no PAN }\end{array}$ & 11 & & & \\
\hline & & 3 & $\begin{array}{l}\text { Promover a mudança na percepção das } \\
\text { populações humanas sobre a importância } \\
\text { biológica de répteis e anfíbios nas áreas } \\
\text { estratégicas do PAN }\end{array}$ & 8 & & & \\
\hline & & 4 & $\begin{array}{l}\text { Ampliar as parcerias entre os órgãos públicos, } \\
\text { setor produtivo e sociedade civil organizada }\end{array}$ & 5 & & & \\
\hline & & 5 & $\begin{array}{l}\text { Reduzir os impactos negativos às espécies } \\
\text { contempladas no PAN causados pelo manejo } \\
\text { inadequado dos recursos naturais }\end{array}$ & 3 & & & \\
\hline \multirow{7}{*}{$\begin{array}{l}\text { PAN } \\
\text { Pequenos } \\
\text { Felinos (2014) }\end{array}$} & \multirow{7}{*}{$\begin{array}{l}\text { Reduzir a } \\
\text { vulnerabilidade de } \\
\text { pequenos felinos } \\
\text { nos diferentes } \\
\text { biomas por meio } \\
\text { da ampliação do } \\
\text { conhecimento } \\
\text { aplicado à } \\
\text { conservação, } \\
\text { da proteção } \\
\text { dos habitat, da } \\
\text { minimização } \\
\text { de conflitos } \\
\text { com atividades } \\
\text { antrópicas e de } \\
\text { ações políticas } \\
\text { efetivas, em cinco } \\
\text { anos }\end{array}$} & 1 & $\begin{array}{l}\text { Compreender como a caça e o abate por } \\
\text { retaliação afetam as diferentes populações de } \\
\text { pequenos felinos em cada bioma, em cinco } \\
\text { anos }\end{array}$ & 6 & \multirow{7}{*}{43} & \multirow{7}{*}{$28 / 28$} & \multirow{7}{*}{$8(28 \%)$} \\
\hline & & 2 & $\begin{array}{l}\text { Reduzir a remoção ilegal (caça, abate, } \\
\text { retirada de animais vivos) de indivíduos das } \\
\text { diferentes populações de pequenos felinos, } \\
\text { em cinco anos }\end{array}$ & 6 & & & \\
\hline & & 3 & $\begin{array}{l}\text { Dimensionar e minimizar os impactos da co- } \\
\text { ocorrência entre pequenos felinos e animais } \\
\text { domésticos e exóticos, em cinco anos }\end{array}$ & 5 & & & \\
\hline & & 4 & $\begin{array}{l}\text { Ampliar o conhecimento sobre os impactos } \\
\text { das doenças na saúde das populações de } \\
\text { pequenos felinos, em cinco anos }\end{array}$ & 3 & & & \\
\hline & & 5 & $\begin{array}{l}\text { Manter e ampliar a conectividade entre } \\
\text { populações de pequenos felinos e reduzir os } \\
\text { processos de fragmentação e perda do habitat } \\
\text { nas suas áreas de ocorrência, considerando os } \\
\text { impactos nas diversas escalas, em cinco anos }\end{array}$ & 6 & & & \\
\hline & & 6 & $\begin{array}{l}\text { Estimular a criação e implementação de } \\
\text { políticas públicas que determinem a redução } \\
\text { da fragmentação e perda de habitat em toda } \\
\text { área de distribuição de pequenos felinos, em } \\
\text { cinco anos }\end{array}$ & 5 & & & \\
\hline & & 7 & $\begin{array}{l}\text { Realizar estudos que avaliem de que forma } \\
\text { os diferentes processos naturais e antrópicos } \\
\text { influenciam a conservação das populações de } \\
\text { pequenos felinos, em cinco anos }\end{array}$ & 12 & & & \\
\hline
\end{tabular}




\begin{tabular}{|c|c|c|c|c|c|c|c|}
\hline PAN (ano) & Objetivo geral & & Objetivos específicos & Ações & $\begin{array}{l}\text { Total } \\
\text { de } \\
\text { ações }\end{array}$ & $\begin{array}{l}\text { Especialistas } \\
\text { no PAN/ } \\
\text { especialistas } \\
\text { contatados }\end{array}$ & $\begin{array}{l}\text { Aceites } \\
\text { para a } \\
\text { pesquisa } \\
(\%)\end{array}$ \\
\hline \multirow{6}{*}{$\begin{array}{l}\text { PAN } \\
\text { Tatu-bola } \\
\text { (2014) }\end{array}$} & \multirow{6}{*}{$\begin{array}{l}\text { Aumentar o } \\
\text { conhecimento } \\
\text { sobre as espécies- } \\
\text { foco e minimizar } \\
\text { o efeito das ações } \\
\text { antrópicas de forma } \\
\text { a contribuir para } \\
\text { a conservação } \\
\text { das espécies de } \\
\text { anfíbios e répteis } \\
\text { contempladas } \\
\text { no PAN da } \\
\text { Mata Atlântica } \\
\text { nordestina, em } \\
\text { cinco anos }\end{array}$} & 1 & $\begin{array}{l}\text { Atualizar as áreas de ocorrência das espécies } \\
\text { (Tolypeutes tricinctus e Tolypeutes matacus) } \\
\text { e avaliar as principais ameaças ao longo de } \\
\text { suas distribuições geográficas }\end{array}$ & 5 & \multirow{6}{*}{38} & \multirow{6}{*}{$27 / 21$} & \multirow{6}{*}{$11(52 \%)$} \\
\hline & & 2 & $\begin{array}{l}\text { Mobilizar as comunidades locais, em áreas } \\
\text { de ocorrência de Tolypeutes tricinctus, } \\
\text { bem como a sociedade em geral, sobre } \\
\text { a importância da proteção da espécie na } \\
\text { Caatinga e no Cerrado }\end{array}$ & 7 & & & \\
\hline & & 3 & $\begin{array}{l}\text { Ampliar o conhecimento sobre a biologia } \\
\text { e ecologia (dinâmica populacional, } \\
\text { variabilidade genética e vulnerabilidade às } \\
\text { alterações antrópicas) para o direcionamento } \\
\text { de estratégias de conservação dos tatus-bola } \\
\text { (Tolypeutes tricinctus e Tolypeutes matacus) }\end{array}$ & 6 & & & \\
\hline & & 4 & $\begin{array}{l}\text { Ampliar, qualificar e integrar a fiscalização } \\
\text { para coibir a caça do tatu-bola (Tolypeutes } \\
\text { tricinctus) }\end{array}$ & 7 & & & \\
\hline & & 5 & $\begin{array}{l}\text { Reduzir a taxa de perda de habitat de } \\
\text { Tolypeutes tricinctus nos próximos cinco anos }\end{array}$ & 10 & & & \\
\hline & & 6 & $\begin{array}{l}\text { Promover a conectividade entre as } \\
\text { populações de Tolypeutes tricinctus nos } \\
\text { próximos cinco anos }\end{array}$ & 3 & & & \\
\hline
\end{tabular}

Biodiversidade Brasileira - BioBrasil.

Fluxo Contínuo

n. 1,2021

http://www.icmbio.gov.br/revistaeletronica/index.php/BioBR

Biodiversidade Brasileira é uma publicação eletrônica científica do Instituto Chico Mendes de

Conservação da Biodiversidade (ICMBio) que tem como objetivo fomentar a discussão e a disseminação de experiências em conservação e manejo, com foco em unidades de conservação $e$ espécies ameaçadas.

ISSN: 2236-2886 